Rev. Adm. Saúde (On-line), São Paulo, v. 19, n. 77: e190, out. - dez. 2019, Epub 20 out. 2019 http://dx.doi.org/10.23973/ras.77.190

ARTIGO ORIGINAL

\title{
Indicadores de qualidade em terapia nutricional: uma revisão integrativa
}

Quality indicators in nutrition therapy: an integrative review

\author{
Thais Ortiz Hammes ${ }^{1}$
}

1. Nutricionista, doutora em gastroenterologia e hepatologia. Nutricionista do Hospital de Clínicas de Porto Alegre, Porto Alegre RS

\section{RESUMO}

Introdução: Indicadores de qualidade em terapia nutricional são ferramentas mensuráveis com habilidade reconhecida para avaliar e acompanhar a efetividade e da aderência aos protocolos e procedimentos assistências em nutrição. O objetivo deste estudo é conhecer os principais indicadores de qualidade de terapia nutricional utilizados na gestão e monitoramento da assistência nutricional por meio de revisão de literatura. Métodos: Foi realizado um levantamento bibliográfico de artigos científicos, no período de 2014 a 2018 publicados nas bases de dados bases MEDLINE e LILACS em conformidade aos descritores em Ciências da Saúde. Resultados: A busca inicial nas bases de dados resultou na seleção de 72 artigos, dos quais 61 foram excluídos por duplicidade ou por não preencherem os critérios de inclusão. Dos 11 artigos selecionados, 8 eram artigos originais e 3 eram revisões. Sete dos artigos incluídos, tiveram como objetivo classificar ou propor indicadores para gestão da qualidade na terapia nutricional. Em sua maioria, sinalizavam para a necessidade de ter uma equipe especializada no atendimento nutricional, realização de avaliação nutricional, indicação e acompanhamento nutricional sistemático e direcionado. Quanto ao resultado da aplicação de indicadores na prática clínica, 3 estudos mostraram a necessidade de adequação de indicadores para infusão de dieta na quantidade adequada, tempo de jejum e ocorrência de diarreia. Dois estudos incluídos avaliaram indicadores em outros cenários que não o ambiente hospitalar e apontavam para o uso de indicadores para triagem, avaliação e acompanhamento nutricional, bem como os 
respectivos registros em prontuário. Conclusão: Os principais indicadores de qualidade na terapia nutricional são relacionados a triagem e avaliação nutricional, indicação precoce e adequada de terapia nutricional, monitoramento da oferta nutricional frente prescrição e acompanhamento de intercorrências relacionada a terapia nutricional.

Palavras-chave: Indicadores de Qualidade em Assistência à Saúde. Terapia Nutricional. Nutrição Enteral. Avaliação Nutricional.

\section{ABSTRACT}

Introduction: Quality indicators in nutrition therapy are measurable tools with recognized ability to assessing and monitoring the effectiveness and adherence to nutrition assistance protocols and procedures. The aim of this study is to know the mainly quality indicators in nutrition therapy used in the management and monitoring of nutritional assistance through a literature review. Methods: $A$ review of scientific articles was carried out from 2014 to 2018, published in the databases MEDLINE and LILACS according to the descriptors in Health Sciences. Results: The initial search in the databases resulted in the selection of 72 studies, of which 61 were excluded for duplicity or did not meet the inclusion criteria. Eight studies were original and 3 were reviews. Seven studies were included aimed to classify or propose quality indicators in the management in nutritional therapy. They indicated the need to have a team specialized in nutritional care and an adequate nutritional assessment and monitoring. Regarding the results of the application of indicators in clinical practice, 3 studies showed that is necessary to optimize the results of indicators for diet infusion in adequate quantity, fasting time and occurrence of diarrhea. Two studies evaluated indicators in other settings than the hospital settings and they pointed out to the use of quality indicators for the screening, evaluation and nutritional monitoring, as well as the registry in medical records. Conclusion: The main quality indicators in nutrition therapy are related to nutritional screening and evaluation, early and adequate indication of nutrition therapy, monitoring of nutritional supply in comparison to the prescription, and monitoring of intercurrences related to nutrition therapy.

Keywords: Quality Indicators in Health Care. Nutritional Therapy. Enteral nutrition. Nutritional Evaluation

\section{INTRODUÇÃO}

A desnutrição é um achado comum em pacientes hospitalizados em todo o mundo e pode ser definida como o estado nutricional no qual a deficiência, excesso ou desequilíbrio de nutrientes causa efeitos adversos no organismo, 
com consequências clínicas e funcionais ${ }^{1,2}$. Estima-se que a desnutrição afete entre $20 \%$ e $60 \%$ dos pacientes hospitalizados sendo associada ao aumento do tempo de permanência hospitalar, incidência de infecções, complicações pós-operatórias, mortalidade e retardo na cicatrização de feridas ${ }^{2,3}$. A assistência nutricional adequada é parte do plano terapêutico do paciente e é capaz de prevenir, minimizar ou reverter os efeitos da desnutrição no ambiente hospitalar ${ }^{4,5}$.

Define-se terapia nutricional (TN) como um conjunto de procedimentos que visa reconstituir ou manter o estado nutricional do indivíduo ${ }^{6}$. A TN na assistência hospitalar deve ser norteada por protocolos institucionais baseados em diretrizes específicas para cada situação6,7. Todos os pacientes em TN devem ser monitorizados rotineiramente a fim de garantir melhores desfechos clínicos e menores custos de internação ${ }^{7}$. Para isso, podem ser utilizados protocolos assistências para direcionar as atividades dos profissionais envolvidos na TN e padronizar as etapas de rastreamento e avaliação nutricional, aquisição de insumos para TN, intervenção nutricional e administração da TN. Estes protocolos também devem prever estratégias para o monitoramento da efetividade, adequação e eventos adversos das TN instituídas ${ }^{5,7}$.

Pensando em garantir a excelência dos serviços prestados à população, foram criados programas de qualidade que visam a utilização de normas para as diversas atividades vinculadas às ações de saúde ${ }^{5,7}$. O controle da qualidade no cuidado em saúde é pautado no uso de indicadores como ferramentas de avaliação. Indicador é uma medida utilizada para determinar, através do tempo, o desempenho de funções, processos e resultados de uma instituição ${ }^{7}$. A avaliação da qualidade da TN pode ocorrer por meio de indicadores de efetividade e resultados, monitoramento de eventos adversos, satisfação do cliente, melhora da qualidade de vida e melhora da relação custo-efetividade ${ }^{7}$. A escolha de indicadores de qualidade adequados é importante para a segurança na tomada de decisões pois indicadores mal selecionados podem aumentar o custo das atividades e direcionar a atenção para etapas que não sejam estratégicas ${ }^{5,6}$.

Em 2017, a Força-Tarefa de Nutrição Clínica do Comitê de Nutrição do International Life Sciences Institute (ILSI) do Brasil realizou uma pesquisa em hospitais brasileiros para avaliar o uso de indicadores de qualidade em TN7. Dentre os 57 hospitais participantes, os indicadores de TN mais comumente utilizados foram volume de nutrição enteral prescrito versus volume infundido; frequência de aplicação da Avaliação Subjetiva Global em pacientes em TN; frequência na medição ou estimativa do gasto energético e necessidade proteica em pacientes em TN; frequência de diarreia em pacientes sob TNE e frequência de jejum por mais de 24 horas. No entanto, um dado preocupante obtido neste estudo foi que $8 \%$ das instituições pesquisadas não utilizavam nenhum indicador de qualidade sendo que a falta de recursos humanos e de apoio operacional foram consideradas as maiores dificuldades para a implementação de indicadores de qualidade em TN pelos hospitais avaliados ${ }^{6}$.

Dessa forma, o objetivo desta revisão é descrever os principais indicadores de qualidade de TN utilizados na gestão e monitoramento da assistência nutricional por meio de revisão de literatura. 


\section{MÉTODOS}

Esta pesquisa trata-se de uma revisão integrativa de literatura. A revisão integrativa da literatura é um método de revisão específico que resume as pesquisas mais relevante sobre um determinado assunto, sejam elas pesquisas empíricas ou teóricas. Este método permite a inclusão de diversos delineamentos de pesquisas para possibilitar uma compreensão mais abrangente de um fenômeno particular ${ }^{8,9}$.

A pesquisa, na revisão integrativa, é realizada por meio de 6 etapas sistematizadas: elaboração da questão de pesquisa, estabelecimento de critérios de inclusão/exclusão de artigos para seleção da amostra, definição das informações a serem extraídas dos artigos selecionados, análise das informações dos estudos incluídos, interpretação dos resultados e apresentação da revisão ${ }^{8,9}$.

\section{Coleta e análise das informações}

A pesquisa de artigos foi realizada nas bases de dados MEDLINE (Medical Literature Analysis and Retrieval System Online) e LILACS (Literatura LatinoAmericana e do Caribe em Ciências da Saúde), utilizando os seguintes descritores:

- Indicadores de qualidade em assistência à saúde (quality indicators, health care),

- terapia nutricional (nutrition therapy),

- nutrição enteral (enteral nutrition) e

- avaliação nutricional (nutrition assessment).

Esses descritores foram combinados e acrescidos do operador booleano "and" na realização da busca na base de dados. As combinações realizadas foram as seguintes:

- indicadores de qualidade em assistência à saúde "and" terapia nutricional;

- $\quad$ indicadores de qualidade em assistência à saúde "and" nutrição enteral;

- indicadores de qualidade em assistência à saúde "and" avaliação nutricional.

Foram incluídos artigos publicados nos últimos 5 anos (de 2014 a 2018) e que apresentassem os textos completos disponíveis em formato digital nos idiomas, português e inglês. A seleção dos estudos ocorreu inicialmente pela leitura dos títulos e resumos. Em seguida, foi realizada a leitura do artigo na integra, avaliando os critérios de elegibilidade já especificados. Os dados extraídos foram transcritos para um instrumento que possibilita o detalhamento de cada estudo ${ }^{9}$. Estes dados foram agrupados e categorizados conforme os resultados e discutidos por categoria. 


\section{RESULTADOS}

A busca inicial nas bases de dados resultou na seleção de 72 artigos (Figura 1), dos quais 69 foram excluídos por duplicidade (24 artigos) ou por não preencherem os critérios de inclusão (37 artigos).

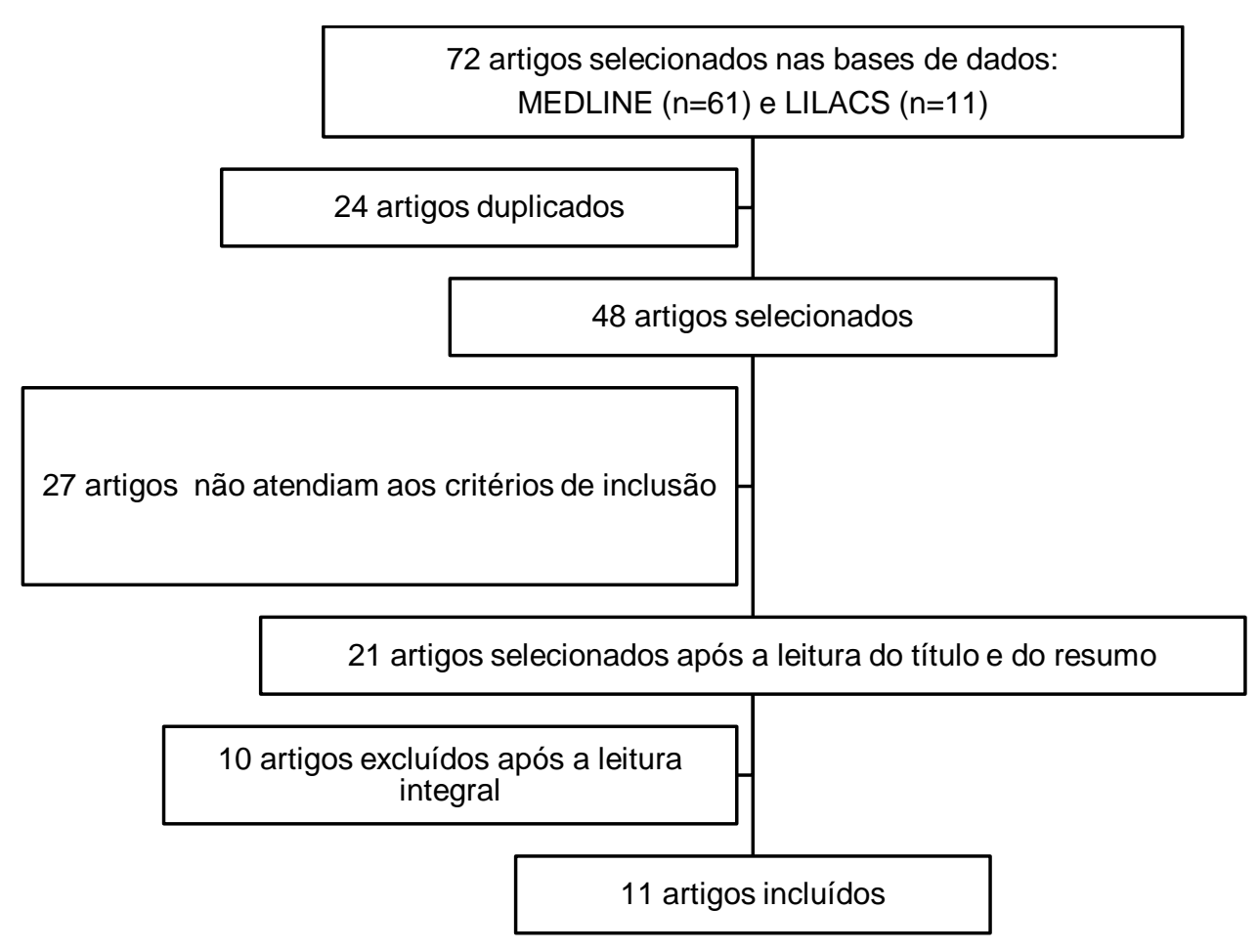

Figura 1. Fluxograma do processo de seleção de artigos nas bases Literatura Latino-Americana e do Caribe em Ciências da Saúde (LILACS) e Medical Literature Analysis and Retrieval System Online (MEDLINE).

Após a leitura analítica destes artigos foram selecionados 11 como objeto de estudo por apresentarem aspectos que respondiam à questão norteadora desta revisão. Destes, 8 eram artigos originais e 4 eram revisões (Tabela 1).

Tabela 1. Principais características dos artigos incluídos.

\section{Título do artigo}

Identifying patient-centered quality indicators for the care of adult home parenteral nutrition (HPN) patients

Indicadores de Qualidade em Terapia Nutricional como ferramenta para

\section{Autores}

Dreesen et al.

\section{Ano de publicação e país Tipo de artigo}

$\begin{array}{ll}2014 & \text { Artigo } \\ \text { Bélgica } & \text { original }\end{array}$

Sá JSM et al. 2014 Artigo de 
avaliação da assistência nutricional em pacientes hospitalizados

Brasil

revisão

Nutrição enteral em pacientes internados

Brandão VL \& 2014

Artigo

em unidade de terapia intensiva: análise

Rosa LPS

Brasil

original

dos indicadores de qualidade

Identification of quality indicators for the nutritional management of adult hospitalized patients by a modified Delphi

Verbanck L et

2015

Artigo

al.

Bélgica

original

process

Desenvolvimento de protótipo de software para auxílio na aplicação de indicadores de qualidade em terapia nutricional

Design of quality indicators for oral nutritional therapy

Lopes SP et

2015

Artigo

al.

Brasil

original

Verotti CCG

2015

Artigo

et al.

Brasil

original

Quality indicators for enteral and parenteral nutrition therapy: application in critically ill patients "at nutritional risk"

Oliveira-Filho

RS et al.

2016

Artigo

Brasil

original

Quality indicators in intensive care

medicine for Germany - third edition 2017

Classificação de indicadores de qualidade em ouro e prata por cenário clínico do serviço público de acordo com

Kumpf $\mathrm{O}$ et al

2017

Artigo de

Alemanha revisão

Santana LS \& 2017

Artigo

Cenicolla GD Brasil

original

especialistas em terapia nutricional

Qualidade da terapia nutricional enteral em unidades de terapia intensiva

Gomes RS et

2017

Artigo

al.

Brasil

original

Process, structural, and outcome quality indicators of nutritional care in nursing

Lorini C et al. 2018

Artigo de

Itália

revisão

\section{DISCUSSÃO}

Indicadores de qualidade na assistência à saúde podem ser entendidos como ferramentas mensuráveis com habilidade reconhecida para avaliar e acompanhar a qualidade do cuidado ${ }^{11}$. A Organização Mundial da Saúde define qualidade como um conjunto de atributos que inclui um nível de excelência profissional, o uso eficiente de recursos, um mínimo de risco ao usuário e um alto grau de satisfação por parte dos clientes ${ }^{5,7}$. Os indicadores de qualidade devem ser ferramentas objetivas, baratas e fácies de aplicar na rotina clínica. 0 uso destes indicadores na assistência nutricional pode ser uma ferramenta útil para o monitoramento da efetividade e da aderência aos protocolos e procedimentos assistências em nutrição ${ }^{12}$. 
A TN pode ser descrita como um conjunto de procedimentos terapêuticos que tem como objetivo de manter ou recuperar o estado nutricional de um indivíduo. Sua implementação pode prevenir ou minimizar algumas consequências da desnutrição como complicações infecciosas, má cicatrização, aumento do tempo de internação e mortalidade ${ }^{13}$. Por isso, o uso sistemático de indicadores de qualidade de TN estabelece uma relação de vigilância e monitoramento em busca de melhores resultados na assistência nutricional do paciente.

Nesta revisão, sete artigos tiveram como objetivo classificar ou propor quais indicadores seriam mais adequados para a gestão da qualidade na TN.

Sá et al. (2014), em revisão da literatura, apresentou e discutiu dez indicadores de TN que haviam sido propostos em outro estudo que contava com a opinião de especialistas em nutrição na escolha destes indicadores ${ }^{13}$. São eles:

- frequência de realização de triagem nutricional em pacientes hospitalizados;

- frequência de diarreia em pacientes sob TNE;

- frequência de saída inadvertida de sonda de nutrição em pacientes em TNE;

- frequência de obstrução da sonda de nutrição em pacientes em TNE;

- frequência de jejum por mais de 24 horas em pacientes em TNE e TNO;

- frequência de pacientes com disfunção da glicemia em TNE e TNP;

- frequência na medição ou estimativa do gasto energético e necessidade proteica em pacientes em TN;

- frequência de infecção de cateter venoso central em pacientes com TNP;

- frequência de conformidade de indicação de TNE;

- frequência de aplicação da Avaliação Subjetiva Global em pacientes em TN.

Já um estudo belga, questionou profissionais envolvidos na TN sobre a aplicabilidade e validade do uso de vários indicadores de qualidade na TN em pacientes hospitalizados ${ }^{14}$. O resultado foi a seleção dos seguintes indicadores, de qualidade por ordem de prioridade:

1) uso precoce de nutrição enteral na ausência de contraindicações;

2) TN em paciente desnutridos ou em risco de desnutrição;

3) ter um formulário para o monitoramento de dietas enterais, nutrição parenteral e suplementos nutricionais;

4) ter uma equipe especializada no suporte nutricional;

5) ter protocolos para a administração de TN;

6) avaliação de triglicerídeos semanal em pacientes com nutrição parenteral;

7) protocolo para administração de medicamentos via sonda nasoentéricas;

8) frequência de reavaliação periódica de pacientes em suporte nutricional;

9) revisão e ajuste frequentes das metas nutricionais por via enteral e parenteral (diário / semanal / duas vezes por semana) e

10) uso amplo de ferramentas de triagem nutricional. 
Ainda, Santana \& Cenicolla (2017), em estudo que ranqueou os indicadores nutricionais, propuseram como classificação "ouro" para terapia intensiva e para pacientes em cuidados de enfermaria, os seguintes indicadores respectivamente:

- frequência de reavaliação periódica em pacientes em TN;

- frequência de medida ou estimativa do gasto energético e necessidades proteicas em pacientes em TN;

- frequência de obstrução de sonda de nutrição em pacientes em TNE;

- frequência de pacientes em TN que atingiram a meta de energia e proteína em $72 \mathrm{~h}$;

- frequência de diarreia em pacientes em TNE e

- frequência de saída inadvertida de sonda de nutrição em pacientes em TNE $^{15}$.

Embora os indicadores propostos nos diferentes estudos sejam textualmente diferentes, se observa que todos versam sobre a mesma temática: ter uma equipe especializada no atendimento nutricional que realize avaliação nutricional, indique e acompanhe de forma sistemática as terapias nutricionais por diferentes vias, tanto no que se refere aos aspectos nutricionais propriamente ditos quanto as implicações práticas das terapias.

Verotti e colaboradores (2015) realizaram um estudo que teve como objetivo avaliar indicadores de qualidade no contexto de terapia nutricional por via oral $(\mathrm{TNO})^{12}$. O resultado apresentou os seguintes indicadores:

- frequência de triagem nutricional;

- frequência de prescrição de suplementação nutricional oral para pacientes desnutridos com dieta via oral;

- frequência de prescrição de TNO para pacientes em risco de desnutrição;

- frequência de avaliação nutricional;

- frequência de adesão a TNO;

- frequência de pacientes internados com ingestão insuficiente e com prescrição do de TNO;

- frequência de avaliação da ingestão oral em pacientes em cuidados intensivos e de enfermaria;

- frequência de intolerância ao volume de 19 suplemento oral devido ao horário de oferta inadequado;

- frequência de intolerância ao sabor e ao volume do suplemento.

O monitoramento de indicadores de TN em pacientes críticos é bastante discutido na literatura. Kumpf e colaboradores propuseram avaliação da nutrição enteral precoce como um indicador de qualidade em cuidados intensivo ${ }^{16}$. No presente estudo 3 artigos avaliaram a adequação da nutrição enteral em pacientes críticos. Brandão \& Rosa (2014) avaliaram o emprego de indicadores de qualidade da TNE em 22 pacientes internados em uma unidade de terapia intensiva ${ }^{17}$. Observou-se inadequação dos seguintes indicadores:

- $\quad 18,2 \%$ para "frequência de jejum digestório por mais de 24 horas", 
- $54,4 \%$ para "frequência de diarreia em pacientes sob TNE" e

- $31,8 \%$ para "frequência de episódios de obstipação em pacientes sob TNE".

Em outro estudo, realizado em uma unidade de terapia intensiva em São Paulo, o uso de 9 indicadores de qualidade de TN foi acompanhado durante 3 anos consecutivos. Os indicadores "frequência de realização de triagem nutricional em pacientes hospitalizados" e "frequência na medição ou estimativa do gasto energético e necessidade proteica em pacientes em TN" foi aplicado em $100 \%$ dos 145 pacientes avaliados. Mais de $70 \%$ da TNE prescrita foi infundida nos 93 pacientes que recebiam nutrição enteral exclusiva e mais de $80 \%$ dos pacientes receberam nutrição enteral precoce. O percentual de inadequação dos indicadores "frequência de saída inadvertida de sonda de nutrição", "frequência de diarreia em pacientes sob TNE" e "frequência de jejum por mais de 24 horas" foi inferior a $10 \%{ }^{18}$.

Em outro estudo também realizado em uma unidade de terapia intensiva, foi avaliada a adequação de calorias e proteínas calculadas, prescritas e ofertadas com como outros indicadores de qualidade da terapia nutricional enteral propostos pelo ILSI do Brasil. Entre os 53 pacientes avaliados, o percentual de adequação entre o prescrito e o infundido para calorias e proteínas foi $71,6 \%$. Foram observadas não conformidades em relação às metas pré-estabelecidas nos seguintes indicadores de qualidade:

- dias com oferta calórica administrada maior ou menor que $20 \%$ da oferta prescrita em pacientes em TNE (25\%),

- dias com aporte proteico insuficiente no total de dias $(49,37 \%)$ e

- pacientes em jejum por mais de 24 horas $(52,83 \%)^{19}$.

Estes estudos evidenciam que, embora os pacientes sejam adequadamente avaliados, ainda existe espaço para otimização da terapia nutricional no que se refere a infusão na quantidade adequada, tempo de jejum e ocorrência de diarreia. Dessa forma, indicadores que abordem estes assuntos devem ser prioritários na assistência nutricional.

A maioria dos estudos encontrados discute o uso de indicadores no contexto de cuidados hospitalares, no entanto, dois estudos avaliaram estas ferramentas em cenários diferentes. Dressen e colaboradores (2014), buscaram encontrar indicadores de qualidade para o cuidado de pacientes adultos com nutrição parenteral domiciliar sob a ótica dos pacientes ${ }^{11}$. Para isso, 20 pacientes foram entrevistados com instrumento semiestruturado. A maioria dos indicadores propostos apontava para os seguintes fatores para atingir as metas nutricionais e necessidade de internação:

- otimização da comunicação com os profissionais de saúde,

- treinamento adequado,

- compartilhamento de experiências entre pacientes,

- qualidade de vida,

- além de indicadores objetivos relacionados ao ganho de peso, sobrevida, ocorrência de infecções e obstruções de cateter. 
Já Lorini et al (2018) em sua revisão sobre indicadores de qualidade no cuidado nutricional em casas de repouso, não encontrou consenso no uso de indicadores de qualidade neste cenário ${ }^{20}$. No entanto, aparecem como indicadores relevantes para avaliação da qualidade deste cuidado nutricional:

- presença de triagem e sua inclusão no prontuário,

- avaliação da perda de peso,

- uso de protocolos de prevenção e tratamento de desnutrição,

- assistência alimentar e

- uso de suplementos alimentar.

Embora um bom indicador deva ser objetivo e fácil de ser obtido, na prática, o que se percebe é que o monitoramento de indicadores de qualidade exige tempo e uma equipe treinada tanto para o registro quanto para o levantamento dos dados. Pensando que sistemas de informação em saúde podem dar suporte, Lopes e colaboradores (2015) desenvolveram um protótipo de software para auxiliar a aplicação de indicadores de qualidade em TN. Apesar de não ter sua usabilidade testada, a ferramenta se apresenta como uma solução interessante para aumentar o uso rotineiro de indicadores por possibilitar o armazenamento e organização de dados clínicos coletados ${ }^{21}$.

Por fim, embora existam diferentes propostas de uso de indicadores de qualidade na TN, pode-se concluir que os mais utilizados estão direcionados para as seguintes áreas da assistência nutricional:

- triagem e avaliação nutricional;

- indicação precoce e adequada de terapia nutricional, monitoramento da oferta frente prescrição e

- acompanhamento de intercorrências relacionada a terapia nutricional (diarreia, glicemia, saída de acesso etc.).

Ainda que a literatura tenha acordo quanto a importância do uso de indicadores de qualidade na TN, mais estudos são necessários a fim de se otimizar as rotinas de monitoramento e a escolha de indicadores adequados para cada contexto.

\section{REFERÊNCIAS}

1. Loser C. Malnutrition in hospital: the clinical and economic implications. Dtsch Arztebl Int [Internet]. 2010 Dec [cited 2019 Sep 25]; 107(51-52): 911-917. Available from: https://www.ncbi.nlm.nih.gov/pmc/articles/PMC3023157/

2. Aquino RC, Philippi ST. Identificação de fatores de risco de desnutrição em pacientes internados. Rev Assoc Med Bras [Internet]. 2011 Dec [cited 2019 Sep 25]; 57(6): 637-643. Available from: http://www.scielo.br/scielo.php?script=sci arttext\&pid=S010442302011000600009\&lng=en. http://dx.doi.org/10.1590/S010442302011000600009. 
3. Correia MI, Hegazi RA, Diaz-Pizarro Graf JI, Gomez-Morales G, Fuentes Gutiérrez C, Goldin MF, et al. Addressing Disease-Related Malnutrition in Healthcare: A Latin American Perspective. JPEN J Parenter Enteral Nutr. 2016;40(3):319-325. DOI: http://dx.doi.org/10.1177/0148607115581373

4. Garcia RWD, Leandro-Merhi VA, Pereira AM. Estado nutricional e sua evolução em pacientes internados em clínica médica. Rev Bras Nutr Clin [Internet]. 2004 [cited 2019 Sep 25]; 19(2):59-63. Available from:

http://citeseerx.ist.psu.edu/viewdoc/download?doi=10.1.1.467.6837\&rep= rep1\&type $=$ pdf\#page $=19$

5. International Life Sciences Institute do Brasil [Internet]. Indicadores de qualidade em terapia nutricional:10 anos de IQTN no Brasil: resultados, desafios e propostas. São Paulo: ILSI Brasil, 2018 [cited 2019 Sep 25]. Available from: http://ilsibrasil.org/wpcontent/uploads/sites/9/2018/06/Fasci\%CC\%81culo-10-anos-de-IQTN$\underline{\text { no-Brasil2-Final.pdf }}$

6. Verotti, CCG. Contribuição para seleção de dez indicadores de qualidade em terapia nutricional. [Dissertação de Mestrado]. São Paulo: Universidade de São Paulo; 2012.

7. Associação Médica Brasileira e Conselho Federal de Medicina: Projeto Diretrizes [Internet]. Terapia Nutricional: Indicadores de Qualidade. São Paulo: Projeto Diretrizes, 2011. [cited 2019 Sep 25]. Available from: https://diretrizes.amb.org.br/ BibliotecaAntiga/terapia nutricional indicad ores de qualidade.pdf

8. Mendes KDS, Silveira RCDCP, Galvão CM. Revisão integrativa: método de pesquisa para a incorporação de evidências na saúde e na enfermagem. Texto contexto - enferm. [Internet]. 2008 Dec [cited 2019 Sep 25]; 17(4): 758-764. Available from: http://www.scielo.br/scielo.php?script=sci arttext\&pid=S010407072008000400018\&lng=en. http://dx.doi.org/10.1590/S0104$\underline{07072008000400018 .}$.

9. Souza EP, Barbosa ECS, Rodrigues A, Leal I, Nogueira LMV. Prevenção e controle da tuberculose: revisão integrativa da literatura. Rev Cuid [Internet]. 2015 July [cited 2019 Sep 26]; 6(2): 1094-1102. Available from: http://www.scielo.org.co/scielo.php?script=sci arttext\&pid=S2216$09732015000200010 \& \operatorname{lng}=$ en or http://dx.doi.org/10.15649/cuidarte.v6i2.178.

10. Ursi, ES. Prevenção de lesões de pele no perioperatório: Revisão integrativa da literatura. [Dissertação de Mestrado]. Ribeirão Preto: Universidade de São Paulo; 2005.

11. Dreesen M, Foulon V, Vanhaecht K, De Pourcq L, Hiele M, Willems L. Identifying Patient Centered Quality Indicators for the Care of Adult Home Parenteral Nutrition (HPN) Patients. JPEN J Parenter Enteral Nutr. 
2014 Sep;38(7):840-6. DOI:

http://dx.doi.org/10.1177/0148607113495891

12. Gimenez Verotti CC, de Miranda Torrinhas RS, Pires Corona L, Waitzberg DL. Design of quality indicators for oral nutritional therapy. Nutr Hosp. 2015 Jun 1;31(6):2692-5. DOI: http://dx.doi.org/10.3305/nh.2015.31.6.8735.

13. Sá, JSM; Marshall, NG. Indicadores de Qualidade em Terapia Nutricional como ferramenta para avaliação da assistência nutricional em pacientes hospitalizados. Com. Ciências Saúde [Internet]. 2014 [cited 2019 Sep 25]; 25(2): 127-140. Available from:

http://bvsms.saude.gov.br/bvs/periodicos/Indicadores de\%20 qualidade em terapia.pdf

14. Verbanck L, De Waele J, Duysburgh I, Van Looy L, Ysebaert D, Merckx $L$ et al. Identification of quality indicators for the nutritional management of adult hospitalized patients by a modified Delphi process. Acta Anaesthesiologica Belgica [Internet]. 2015 [cited 2019 Sep 25];66(4):1-8. Available from: https://www.sarb.be/site/assets/files/1142/01verbanck et al.pdf

15. Santana LS, Ceniccola GD. Classificação de indicadores de qualidade em ouro e prata por cenário clínico do serviço público de acordo com especialistas em terapia nutricional. Braspen J [Internet]. 2017 [cited 2019 Sep 25]; 32 (4): 369-74. Available from:

http://arquivos.braspen.org/journal/out-dez-2017/12-Classificacao-deindicadores.pdf

16. Kumpf O, Braun JP, Brinkmann A, Bause $\mathrm{H}$, Bellgardt $\mathrm{M}$, Bloos $\mathrm{F}$ et al. Quality indicators in intensive care medicine for Germany - third edition 2017. Ger Med Sci. 2017 Aug 1;15: Doc10. DOI: http://dx.doi.org/10.3205/000251

17. Brandão VL, Rosa LPS. Nutrição enteral em pacientes internados em unidade de terapia intensiva: análise dos indicadores de qualidade. Brasília méd. 2014; 50(3). DOI: http://dx.doi.org/10.14242/2236$\underline{5117.2014 a v 50 n 3 a 90 p 200}$

18. Oliveira Filho RS, Ribeiro LM, Caruso L, Lima PA, Damasceno NR, García Soriano F. Quality indicators for enteral and parenteral nutrition therapy: application in critically ill patients "at nutritional risk". Nutr Hosp. 2016 Sep 20;33(5):563. DOI: http://dx.doi.org/10.20960/nh.563.

19. Gomes RS, Cabral NAL, Oliveira ATV. Qualidade da terapia nutricional enteral em unidades de terapia intensiva. Braspen J [Internet]. 2017 [cited 2019 Sep 25]; 32 (2): 165-9. Available from: http://www.braspen.com.br/home/wp-content/uploads/2017/08/13-AOQualidade-da-terapia-nutricional.pdf 
20. Lorini C, Porchia BR, Pieralli F, Bonaccorsi G. Process, structural, and outcome quality indicators of nutritional care in nursing homes: a systematic review. BMC Health Serv Res. 2018 Jan 26;18(1):43. DOI: http://dx.doi.org/10.1186/s12913-018-2828-0.

21. Lopes SP, Castro MG, Vasconcelos MIL, Lopes JP, Oliveira JF. Desenvolvimento de protótipo de software para auxílio na aplicação de indicadores de qualidade em terapia nutricional. J. Health Inform [Internet]. 2015 Jul-Set [cited 2019 Sep 25]; 7(3): 75-81. Available from: http://www.jhi-sbis.saude.ws/ojs-jhi/index.php//hi-sbis/article/view/341

Recebido: 05 de outubro de 2019. Aceito: 17 de outubro de 2019

Correspondência: Thais Ortiz Hammes. Rua Ramiro Barcelos, 2350. CEP 90035-903 - Porto Alegre, RS E-mail: thaisortizhammes@gmail.com

Conflito de Interesses: os autores declararam não haver conflito de interesses.

(C) This is an Open Access article distributed under the terms of the Creative Commons Attribution License, which permits unrestricted use, distribution, and reproduction in any medium, provided the original work is properly cited 